\title{
Study of the structure of purulent-inflammatory disease incidence at lower jaw fracture
}

\author{
Abdullaev A.Kh. \\ Tashkent State Dental Institute, Tashkent city Department of Maxillofacial Surgery \\ Email address: \\ akom_doc@inbox.ru (AbDullaev A.Кн.)
}

To cite this article:

Abdullaev A.Kн. Study of the structure of purulent-inflammatory disease incidence at lower jaw fracture. Journal of research in health science. Vol. 2, No. 2, 2017, pp. 8-11. DOI 10.26739/2523-1243

\section{doi http://dx.doi.org/10.26739/2523-1243/-2017-2-2-2}

\begin{abstract}
: the study analyzed 94 cases of patients with a fracture of the lower jaw complicated by infectious-inflammatory disease, especially traumatic osteomyelitis of the lower jaw. Analysis of purulentinflammatory processes in the maxillofacial area showed occurrence of osteophlegmon, osteoabscess, traumatic osteomyelitis of the lower jaw.
\end{abstract}

Keywords: purulent-inflammatory process, maxillofacial area, lower jaw fracture, odontogenic osteomyelitis, sequestrectomy operation.

The incidence of purulentinflammatory processes in the maxillofacial area at the present stage does not tend to decrease. The specific weight of hospitalization of patients with purulent-inflammatory diseases of the maxillofacial area is from 27.2 to $61 \%$. The most common complication of fractures of the lower jaw is traumatic osteomyelitis. According to various authors, the frequency of it reaches $10-30 \%$ [2].

Along with treatment of destructive forms of odontogenic osteomyelitis of the jaws, prevention and treatment of infectious and inflammatory complications of facial bones fractures, mainly traumatic osteomyelitis, are topical problems of contemporary clinical maxillofacial surgery $[1,4,5,6,7]$.

The aim of the research - to study the structure of the disease incidence of traumatic osteomyelitis in the period from 2010 to 2016 years to prevent its developing after fractures of the lower jaw.

Materials and methods. A retrospective analysis of 94 case histories of patients of different age groups, with a fracture of the lower jaw complicated by infectious and inflammatory disease, especially 
traumatic osteomyelitis of the lower jaw, treated at the department of Adults ' Maxillofacial Surgery of the Tashkent State Dental Institute in the period from 2010 to 2016 was made. Cases of infectious-inflammatory complications after the fracture of the lower jaw were registered owing to the fact that suppuration of soft tissues in the area of damage is one of the causes of traumatic osteomyelitis of the lower jaw. Moreover, the infectious-inflammatory process of soft tissues can lead to infection of the bone wound and inflammation of the bone marrow.

In analysis of the case histories of patients, the evaluation criteria were: age, sex, term of visit, diagnosis, period of hospitalization, etiology, anatomical area of spreading, type of surgical treatment. The received data were subjected to statistical processing using the application package Microsoft Excel.

\section{Results and discussion.}

The analysis of archival material showed that the greatest number of registered patients with fracture of the lower jaw complicated by infectious and inflammatory disease as well as traumatic osteomyelitis of the lower jaw was observed in $2012(\mathrm{n}=21)$, the smallest in $2014(\mathrm{n}=5)$ (Fig. 1).

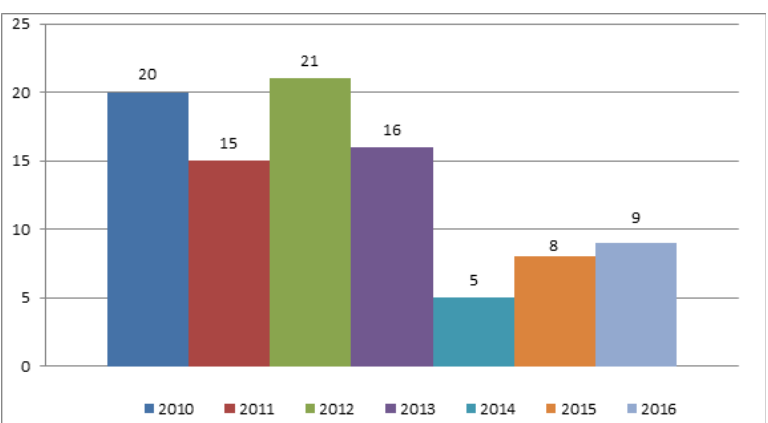

Fig. 1. Distribution of patients from 2010 to 2016.
It was determined that the age of patients varied from 19 to 62 years, and the average age was $36.4 \pm 1.3$ years. The greatest number of patients was ablebodied age of 23 to 37 years. Among the patients, a greater percentage of trauma was observed in men $88 \%(\mathrm{n}=83)$ than in women - $12 \%(\mathrm{n}=11)$.

Among all the patients with a fracture of the lower jaw, the incidence of soft tissue infection (osteogenic phlegmon) was 58\%, while the osteogenic abscess was $37.8 \%$ (Fig. 2). Moreover, among the anatomical regions of the soft tissues involved in osteophlegmon and osteoabscess, the submandibular region prevailed, in $60.7 \%$ and $50 \%$ of cases, respectively. The number of cases of osteophlegmon occupying two anatomical regions was about $30.2 \%$, three - about $7 \%$ of the total, moreover, the cases of associated involvement in the purulent inflammatory process of submandibular and parotid-chewing regions (38\%) prevailed. The number of associated topographic and anatomical regions affected the hospitalization period of patients, so in the presence of a purulent-inflammatory process in two anatomical regions, the average period of hospitalization was $3.6 \pm 0.4$ days, and with the involvement of three regions in the process, $4.7 \pm 0.9$ days. In addition, the average period of hospitalization was affected by the number of performed operations, so in cases of reoperation the average period of hospitalization of the patient increased by 2.4 times and in average composed $7.7 \pm 0.3$ days.

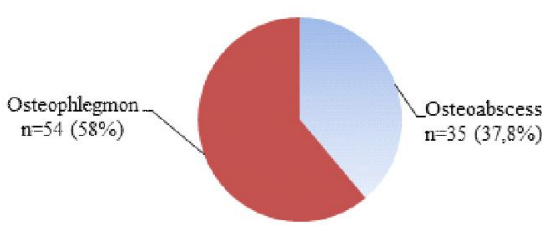

Fig. 2. The ratio of occurrence of osteoabscesses and osteophlegmons. 
Abdullaev A.Kh. Study of the structure of purulent-inflammatory disease incidence at lower jaw fracture.

With a fracture of the lower jaw, one of the causes of inflammation is the presence of a tooth in the fracture line [3]. According to the literature, $67-82 \%$ of the fractures of the lower jaw, localized within the dentition, are open and so, are considered to be primarily complicated due to bone wound infection with a pathogenic microflora (E.Kruger, 1986). Moreover, with fractures of the lower jaw due to the presence of immobilizing wiring, the process of selfcleaning of the oral cavity sharply disrupts, leading to an increase in the number of pathogenic microorganisms on the surface of the teeth and mucous membrane, increasing the probability of infection of the bone wound (AI Kaspina, 1981; 1985; Adzhiev KS, 1991).

Thus, the study showed that during the period of the disease from the moment of trauma to the 21st day, among the patients with an infectious-inflammatory complication of the fracture of the lower jaw, the number of osteophlegmons $(63.8 \%)$ with a tooth in the fracture line prevailed - in $75.9 \%$ of cases. Timely opening of the purulent focus, removal of the tooth from the fracture line, adequate immobilization of bone fragments and providing antibacterial, detoxification and general restorative therapy helped to stop the inflammatory process without manifestation of clinical signs of necrosis of bone tissue.

Three weeks after injury, 4 patients had osteoabscess of soft tissues in the area of the lower jaw fracture with the presence of a tooth in the fracture line. In 5 clinical cases, the development of osteophlegmons was observed, in $60 \%$ of which there was a presence of a tooth in the fracture line, as the cause of inflammation.
In 7 male patients the traumatic osteomyelitis of the lower jaw was observed, the majority of which came for specialized medical care late (after about 1.5 months), because of living in remote regions of the Republic.

In our study, we noted a clinical case of a patient hospitalized on the 4 th day after injury, in which a fracture of the lower jaw, the presence of a tooth in the fracture line, and an inflammatory complication in the form of osteophlegmon of the parotid-chewing region were observed. The term of hospitalization of this patient corresponded to the acute stage of traumatic osteomyelitis, as this stage is well masked with common inflammatory process in soft tissues, and bone marrow inflammation at the ends of bone fragments due to insignificant volume and absence of reliable clinical signs is not diagnosed. As a result, after 1.5-2 months, which corresponded to the period of development of the chronic stage, this patient was repeatedly hospitalized with a diagnosis of traumatic osteomyelitis of the lower jaw.

In all patients, the average size of the sequestrum was about $0.7 \mathrm{~cm}$. Of the concomitant common diseases cases of mild and moderate anemia, type II diabetes and chronic hepatitis $C$ have been reported. Effective local treatment of these patients consisted of providing the sequestrectomy operation, removal of the tooth closely located to the bone destruction zone, complete immobilization of bone fragments with arch bars and wire suture.

Thus, as a result of the study, it can be concluded that the development of traumatic osteomyelitis of the lower jaw 
is facilitated by: late treatment of the patient in a hospital; the presence of teeth (roots) in the line of the fracture with the formation of inflammatory complication of the perimaxillary soft tissues.

Conclusion.
The results of the analysis of clinical course of purulent-inflammatory diseases with fracture of the lower jaw, specifically traumatic osteomyelitis of the lower jaw can be used in developing of the ways to improve the prevention and treatment of this disease.

\section{References}

[1] Datsko AA, Tetyukhin DV Implementation of modern principles of treatment of injuries of the maxillofacial area / / Stomatology. - 2003. - No. 1. - P. 17-22.

[2] Yefimov Y.V. Fractures of the lower jaw and their complications: medical dissertation. - M., 2004. - $287 \mathrm{p}$.

[3] Zhilov AA, Abdukhalik-Zade N. Sh. Open fractures of the lower jaw // Problems of Biology and Medicine. Samarkand, 2015, No. 1 (82). - P.167-170.

[4] Ivanuta I.V. Optimization of the process of reparative osteogenesis in the treatment of patients with fractures of the lower jaw: Phd dissertation. Stavropol, 2006. - 25 p.

[5] Kirpichnikov M.V., Fomichev E.V., Yarygina E.N., Fomichev D.E. Etiology and clinic of atypical and chronic inflammatory diseases of the maxillofacial region // Journal Volgograd medical university. - 2003. - Vol. 9. - P. 187-189.

[6] Ida M., Watanabe H., Tetsumura A., Kurabayashi T. CT findings as a significant predictive factor for the curability of mandibular osteomyelitis: multivariate analysis // Dentomaxillofac. Radiol. - 2005. - V. 34, No. 2. - P. 86-90.

[7] Scolozzi P., Lombardi T., Edney T., Jaques B. Enteric bacteria mandibular osteomyelitis // Oral Surg., Oral Med., Oral Pathol., Oral Radiol., Endodont. - 2005.-V.. 99, No. 6. - P. 42-46. 